\title{
Comparison of Amniotic Fluid Index and Single Deepest Vertical Pool method for predicting fetal outcome
}

\author{
Rajiv Shah, Paban Sharma \\ Department of Obstetrics and Gynecology, ${ }^{1}$ Karnali Academy of Health Sciences , ${ }^{2}$ Patan Academy of \\ Health Sciences
}

\section{Correspondence}

Dr Rajiv Shah

Karnali Academy of Health

Sciences, Jumla, Nepal

Email:

rjvshaj512@gmail.com

DOI: http://dx.doi.org/10.3126/ jemsn.v13i4.16893

Orcid ID: orcid.org/0000-0002 $-6478-8111$

Article received: Mar $4^{\text {th }} 2017$ Article accepted: Dec $18^{\text {th }} 2017$

\begin{abstract}
Background \& Objectives: The measurement of amniotic fluid volume (AFV) has been an important component of antenatal evaluation of fetal well being. The most commonly used methods are by amniotic fluid index technique (AFI) and single deepest pool method (SDVP). Both the methods employ ultrasound for AFV measurement. The objective of this study is to compare the usefulness of AFI and SDVP method in assessing amniotic fluid volume for predicting adverse perinatal outcome. Materials \& Methods: This is a hospital based prospective comparative study. The patient whose amniotic fluid was measured by SDVP technique was study group and that by AFI was comparison group. Any case with high risk factor was excluded from the study. First case was decided by lottery and then alternately one case was kept in study group and the other in comparative group. If the women did not deliver within 1 week the measurements was retaken and the final values was used for analysis.AFI was estimated as described by Phelan and colleagues and SDVP as described by Manning. Data collection was started after the approval of Institution review board. This study was conducted at Patan Academy of Health Sciences. Duration of data collection was 3 months, Asadh -Bhadra, 2071. Results: A total of one hundred and fifty four cases which met the inclusion criteria were taken. Incidence of oligohydramnios by SDVP method was $10.4 \%$ by SDVP technique and by AFI method it was $18.2 \%$. There was no statistical significant difference between the two groups in terms of rate of induction, mode of delivery, meconium staining of liquor, fetal heart rate tracings, APGAR score at 5 mins and admission to special care baby unit. Conclusion: In non-high risk pregnancy AFI technique detects slightly more number of oligohydramnios as compared to SDVP technique without apparently any difference in perinatal outcome.
\end{abstract}

Key words: Amniotic Fluid Index (AFI); Oligohydramnios; Single deepest Pool Method (SDVP)

Citation: Shah R. Comparison of Amniotic Fluid Index and Single Deepest Vertical Pool method for predicting fetal outcome. JCMS Nepal. 2017;13(4):401-5-22.

\section{INTRODUCTION}

Volume of amniotic fluid (AFV) is measured by ultrasound in two ways by SDVP method and by AFI technique. Diminished amniotic fluid is termed as oligohydramnios. It is arbitrarily defined as AFI $<5 \mathrm{~cm}$ or SDVP $<2 \mathrm{~cm}$. AFV influences the fetal outcome and interventions during pregnancy. Women with oligohydramnios had significantly increased 2.2 fold risk for caesarean delivery for fetal distress and 5.2 fold increased for a five minute APGAR score of less than seven, more chances of cord compression ,fetal heart rate declerations and meconium stained liquor ${ }^{1}$ There is no evidence suggesting that one method was superior to other in the prediction of adverse perinatal outcome. ${ }^{2}$ Hence, this study tries to compare between the two commonly used ultrasonic method of amniotic fluid estimation for predicting fetal outcome.

\section{MATERIALS AND METHODS}

This is a hospital based prospective comparative 
study done in PAHS, between Asadh -Bhadra 2071. Prior to data collection ethical clearance was taken from IRB. Women whose amniotic fluid volume was measured by using SDVP method was study group and by AFI was comparison group. All the cases meeting the inclusion criteria were selected for the study. First case was decided by lottery and then alternately one case was kept in study group and the other in comparative group. A total of 154 cases were taken with 77 in each group. Singleton pregnancy of $>37$ weeks with cephalic presentation was included in this study. All the cases with associated hypertension, diabetes, and congenital anomaly, multiple pregnancies, previous caesarean delivery, fetal malpresentations and malpositions, premature rupture of membrane and antepartum haemorrhage were excluded from the study.

\section{Amniotic Fluid Measurement}

After informed consent was taken, the participant was placed in the supine position and the sonologist estimated AFV by AFI in one group and SDVP in other group. The AFI was estimated by the four quadrant method as described by Phelan and colleagues. ${ }^{3}$ For measuring SDVP the image of the deepest cord free pool pocket was frozen and measured along its maximum length in centimetres as described by Manning and colleagues. ${ }^{4}$ If the women did not deliver within one week the measurements was retaken and the final values were used for analysis. Oligohydramnios was defined by AFI of $5 \mathrm{~cm}$ or less and SDVP of $2 \mathrm{~cm}$ or less. Cardiotocography was performed in all the cases. Fetal distress was diagnosed when a CTG showed significant variable decelerations or persistent late decelerations in the fetal heart rate or prolonged bradycardia, or when the APGAR score was less than seven at five minutes, or when there was meconium stained liquor.

\section{Data Collection}

Data collection was started after the approval of Institution review board. All the fellow doctors, nursing staff and other concerned persons were oriented regarding the protocol of the study. Data was collected 24 hours a day twice in a week and in rest of the days from 8 am to $5 \mathrm{pm}$ by researcher himself. In the remaining hours he took the help from his colleague doctors and nursing staffs. Cases were enrolled from antenatal wards that fulfil the exclusion and inclusion criteria. Written informed consent was taken for enrolment of the cases. All these enrolled cases were followed up throughout their hospital stay. At the end a conclusion was drawn depending upon the findings of the study. Test of significance was done by Chi-square tests by using SPSS 16 .

\section{RESULTS}

During the study period of three months, a total of one hundred and fifty four cases of oligohydramnios which met the inclusion criteria were taken. Incidence of oligohydramnios by SDVP method was $10.4 \%$ by SDVP technique and by AFI method it was $18.2 \%$. In the SDVP group 23 patients $(29.9 \%)$ had induction of labor and in the AFI group $25(32.5 \%)$ patients had induction of labor (P-value $=0.728)$. In the SDVP group 12 $(15.6 \%)$ had fetal distress and in the AFI group 10 $(13 \%)$ had fetal distress $(\mathrm{P}$-value $=0.645)$. There were $6(7.8 \%)$ number of admissions in the SDVP group and $5(6.5 \%)$ in the AFI group (Pvalue $=0.754)$. There was meconium staining of liquor in $21(27.3 \%)$ patients in the SDVP group and $15(19.5 \%)$ patients in the AFI group (P-

Table No. 1: Findings in the patients

\begin{tabular}{llll} 
Variables & SDVP & AFI & p-value \\
\hline Incidence of oligohydramnios & $8(10.4 \%)$ & $14(18.2 \%)$ & 0.15 \\
\hline Induction of labor & $23(29.9 \%)$ & $25(32.5 \%)$ & 0.728 \\
Fetal distress & $12(15.6 \%)$ & $10(13 \%)$ & 0.645 \\
\hline $\begin{array}{l}\text { Admission in special care baby } \\
\text { unit }\end{array}$ & $6(7.8 \%)$ & $5(6.5 \%)$ & 0.754 \\
Meconium stained liquor & $21(27.3 \%)$ & $15(19.5 \%)$ & 0.253 \\
Abnormal CTG findings & $7(9.1 \%)$ & $10(13 \%)$ & 0.44 \\
Instrumental or caesarean delivery & $21(27.3 \%)$ & $19(24.7 \%)$ & 0.53 \\
$\begin{array}{l}\text { APGAR score of less than 7 at 5 } \\
\text { mins }\end{array}$ & $4(5.2 \%)$ & $2(2.6 \%)$ & 0.67 \\
SDVP-single deepest vertical method, AFI- amniotic fluid index, CTG-cardiotocography & \\
\hline
\end{tabular}


value $=0.253)$. There were $7(9.1 \%)$ non-reactive CTG in the SDVP group and $10(13 \%)$ in the AFI group $(p$-value $=0.440)$. There were $21(27.3 \%)$ abnormal deliveries in the SDVP group and 19 $(24.7 \%)$ in the AFI group (p-value $=0.523$ ). APGAR score at 5 min of less than seven was 5.2 $\%$ in the SDVP group and $2.6 \%$ in the AFI group $(p-v a l u e=0.677) .($ See table $)$

\section{DISCUSSION}

Amniotic fluid volume serves as an indicator of fetal well being. Decreased amniotic fluid volume is associated with fetal congenital anomalies, post maturity syndrome, IUGR, and increased perinatal morbidity and mortality. Amniotic fluid volume highly influences the fetal outcome. The evaluation of the amniotic fluid volume has been an integral component of the fetoplacental assessment.

In my study the incidence of oligohydramnios was $18.2 \%$ by AFI method and $10.4 \%$ by SDVP method. In the study by Moses et $\mathrm{al}^{12}$ the incidence of oligohydramnios was $25 \%$ by AFI method and $8 \%$ by SDVP method. In another study by Chauhan et $\mathrm{al}^{13}$ the incidence of oligohydramnios was $17 \%$ by AFI method and $10 \%$ by SDVP method. These findings are similar to the present study. But the incidence of oligohydramnios in the study by Dasari et al 14 was $34 \%$ by AFI method and $59 \%$ by SDVP method and in the study by Miyamura et $a{ }^{15},{ }^{15}$ the incidence of oligohydramnios by SDVP method was $19 \%$ and by AFI was $30.5 \%$. These studies measured AFV only in postdated pregnancies. So, incidence is higher in these studies. In the studies by Alfirevic et al, ${ }^{16}$ Myles et $\mathrm{al}^{3}$ and Morris et $\mathrm{al}^{8}{ }^{8}$ the incidence of oligohydramnios by AFI was between $7.9 \%$ to $10 \%$ and by SDVP was between $1.4 \%$ to $2.4 \%$. The incidence of oligohydramnios was higher in the present study as compared to other studies, possibly due to more frequent amniotic fluid measurement, i.e. twice weekly after 40 weeks of gestation. In most of these studies the incidence of oligohydramnios is more in AFI group as compared to SDVP group which is also true in my study. The incidence of oligohydramnios by both methods is very high in the studies which has taken only post dated pregnancies.

Rate of induction of labor in SDVP group was $29.9 \%$ and in the AFI group was $32.5 \%$. There is slightly more number of inductions in AFI group but it is not statistically significant as P-Value is 0.728. In the study by Chauhan et $\mathrm{al}^{13}$ the rate of induction of labor in AFI group was $24 \%$ and in the SDVP group was $21 \%$ but it was not statistically significant. In the study by Moses et $\mathrm{al}^{12}$ the rate of induction of labour was $19 \%$ in both SDVP and AFI group. In another study by Alfiveric et $\mathrm{al}^{16}$ the induction of labor was in $34.8 \%$ cases in AFI group and $30.8 \%$ in SDVP group. This was statistically significant. Similarly, there was statistically significant increase in the rate of inductions in the AFI group as compared to SDVP group in the studies by Magann et al, ${ }^{17}$ Verrotti et al, ${ }^{9}$ Moore et al, ${ }^{18}$ and Dassari et al. ${ }^{14}$ And there was no significant difference in the rate of inductions in the other studies by Miyamura et $\mathrm{al}^{15}$ and Moses et al ${ }^{12}$ including my study.

In the present study the rate of fetal distress was $15.6 \%$ in SDVP group and 13\% in AFI group. This was not statistically significant as the P-value was $0.645 \%$. Similarly, non reactive CTG findings were present in $9.1 \%(\mathrm{n}=7)$ in SDVP group and $13 \%$ $(n=10)$ in AFI group. But it was also not statistically significant between the two groups as the P- Value calculated was 0.440 . Similarly, the meconium staining of liquor was $27.3 \%(n=21)$ in the SDVP group and $19.5 \%(n=15)$ in AFI group. This too was statistically non-significant. Similar findings were found in the study by Alfirevic et al, ${ }^{16}$ Chauhan et al,${ }^{13}$ Magann et al ${ }^{17}$ and Moses et al. ${ }^{12}$ In these studies there was no significant difference between the two group in the occurrence of fetal distress, abnormal CTG patterns and meconium staining of liquor between the groups. While in the study by Miyamura et al, ${ }^{15}$ Myles et al ${ }^{3}$ and Verrotti et al, ${ }^{9}$ the occurrence of abnormal CTG pattern, fetal distress and meconium staining of liquor was more in the SDVP group as compared to AFI group and this was statisitically significant thus concluding that SDVP was better than AFI in predicting adverse fetal outcome. While in the study by Youssef et $\mathrm{al},{ }^{8}$ Fisher et $\mathrm{al},{ }^{4}$ and Morris et $a l^{5}$ the occurrence of fetal distress, meconium staining of liquor and abnormal CTG pattern was significantly higher in the AFI group as compared to SDVP group. But in these studies the subject were post term pregnancy and thus the conclusion made from these studies was that in post term pregnancy AFI was better predictor of adverse fetal outcome as compared to SDVP technique.

Regarding mode of delivery in the present study, $22.1 \%(\mathrm{n}=17)$ had caesarean delivery, $5.2 \%(\mathrm{n}=4)$ had instrumental delivery and $72.7 \%(\mathrm{n}=56)$ had normal vaginal delivery in the SDVP group. And in 
the AFI group $23.4 \% \quad(n=18)$ had caesarean delivery and $1.3 \%(\mathrm{n}=1)$ had instrumental delivery and $75.3 \%(n=58)$ had normal vaginal delivery. There was no statistical difference between the two groups in terms of caesarean delivery and instrumental delivery. Similar to the present study, there was no difference in the rate of caesarean delivery and the instrumental delivery between the two groups in the study by Alfirevic et al, ${ }^{16}$ Myles et al, ${ }^{3}$ Fisher et al, ${ }^{8}$ and Morris et al. ${ }^{5}$ And in the study by Magann et $\mathrm{al}^{17}{ }^{17}$ Youssef et $\mathrm{al}^{4}$ and Chauhan et $\mathrm{al}^{13}{ }^{13}$ there was higher incidence of caesarean section and instrumental delivery in the SDVP group as compared to the AFI group. But in the study by Miyamura et $\mathrm{al}^{15}$ and Moses et $\mathrm{al}^{12}$ there was higher incidence of caesarean delivery and instrumental delivery in the AFI group as compared to the SDVP group. These studies showed variable results and superiority of one method over the other cannot be established.

Admission to the special care baby unit was $7.8 \%$ $(n=6)$ in SDVP group and 6.5\% $(n=5)$ in the AFI group. This was also not statistically significant in between the groups. Similarly APGAR score at five minutes of less than seven was $5.2 \%$ in SDVP group and $2.6 \%$ in AFI group. This value is not statistically significant as p-value is 0.677 . There were no neonatal mortality in the either group. There were no significant difference in the rate of admission to special care baby unit and lower APGAR score at five minutes in the either group in the studies by Alfirevic et al, ${ }^{16}$ Chauhan et al, ${ }^{13}$ Magann et al, ${ }^{19}$ Moses et al, ${ }^{12}$ Verrotti et al, ${ }^{9}$ Moore et al, ${ }^{18}$ and Dassari et al, ${ }^{14}$ similar to this study.

Hence, from the present study we can interpret that there was more incidence of oligohydramnios in the AFI group as compared to SDVP group without difference in the rate of induction of labor, occurrence of fetal distress, admission to special care baby unit, nonreactive CTG pattern, meconium staining of liquor, APGAR score of less than seven at five minutes and mode of delivery.

\section{CONCLUSION}

From our study we conclude that in non-high risk pregnancy, AFI technique detects slightly more number of oligohydramnios as compared to SDVP technique without apparently any difference in perinatal outcome in terms of occurrence of fetal distress, abnormal CTG findings, mode of delivery, low APGAR score at five minutes and admission of neonates to special care baby unit.

\section{LIMITATIONS OF THE STUDY}

The sample size of our study was very small and the duration of the study was also only three months. And also more accurate result would have come if the outcome was measured only in oligohydramnios patients of the either group.

\section{ACKNOWLEDGEMENTS:}

I would like to acknowledge Professor $\mathrm{Dr}$ Gehanath Baral and Mrs Amita Shrestha for their invaluable guidance while conducting this research.

\section{REFERENCES}

1. Oosterhoof H, Haak MC, Arnoudes JG, Acute Maternal Rehydration increases the urine production rate in the near term human fetus. American Journal of Obstetrics and Gynecology. 2000;183:226-9. https://doi.org/10.1016/ S0002-9378(00)17852-4. PMID: 10920336.

2. Nabhan A, Abdelmoula YA. Amniotic fluid index versus single deepest vertical pocket: a metaanalysis of randomized controlled trials. International Journal of Gynecol and Obstet. 2009;104:184-8. https:// doi.org/10.1016/j.ijgo.2008.10.018. PMID: 19046586.

3. Myles TD, Santolaya -Forgas J. Normal ultrasonic evaluation of amniotic fluid volume in low-risk patients at term. J Reprod Med. 2002;47:621-4. PMID: 12216427.

4. Youssef A, Abdulla SH, Sayed EH, Salem H, Abdullahim AM, Devoe LD: Superiority of amniotic fluid index over amniotic fluid pocket measurement for predicting bad fetal outcome. South Med J. 1993;86:426-9. https:// doi.org/10.1097/00007611-199304000-00011.

5. Fischer RL, Mc Donell M, Bianculli KW, Perry RL, Hedinger ML, Scholl TO. Amniotic fluid volume estimation in postdate pregnancy: A comparison of techniques. Obstet gynecol. 1993;81:698-704. PMID: 8469457.

6. Magann EF, Chauhan SP, Martin JN jr. Is the amniotic fluid volume predictive of fetal acidosis at delivery? Aust NZ J Obstet Gynaecol. 2003;43:129-33. https:// doi.org/10.1046/j.0004-8666.2003.00042.x. PMID: 14712968.

7. Ajayi RA, Soothill PW. Ultrasound assessment of amniotic fluid volume: a comparison of the single deepest pool and amniotic fluid index to predict perinatal morbidity. Ultrasound Obstet Gynecol. 1991;1:401-404. https:// doi.org/10.1046/j.1469-0705.1991.01060401.x. PMID: 12797022 .

8. Morris JM, Thompson K, Smithey J. The Usefulness of ultrasound assessment of amniotic fluid in predicting adverse outcome in prolonged pregnancy; a prospective double blinded observational study. BJOG. 2003;110:98994. https://doi.org/10.1111/j.1471-0528.2003.02417.x. PMID: 14592583 .

9. Verrotti C, Bedochhi L, Piantelli G, Cavallotti D, Fiene S, Gramellin D. Amniotic Fluid index versus largest vertical pocket in the prediction of perinatal outcome in post term non high risk pregnancies, Acta Biomed. 2004;75 Suppl 1 67-70. PMID: 15301295.

10. Phelan JP, Ahn MO, Smith CV, Rutherford CV, Anderson E. Amniotic Fluid index measurements during pregnancy, J Reproductive Med. 1987;32:601-4. PMID: 3309290.

11. Manning FA, Baskett TF, Morrison I, Lange I.Fetal biophysical profile scoring; A prospective study in 1,184 high risk patients. Am J Obstet Gynecol. 1981;140:289-94. https://doi.org/10.1016/0002-9378(81)90275-1.

12. Moses J, Doherty D, Magann EF, Chauhan SP, Morrison JC. A randomised clinical trial of the intrapartum 
assessment of amniotic fluid volume: Amniotic fluid index versus the single deepest pocket technique. Am J Obstet Gynecol. 2004;190:1564-69. https://doi.org/10.1016/ j.ajog.2004.03.046. PMID: 15284736

13. Chauhan SP, Hendrix NW, orison JC, Magann EF, Devoe LD. Intrapartum Oligohydramnios does not predict adverse peripartum outcome among high risk parturients. Am J Obstet gynecol. 1997;176:1130-38. https://doi.org/10.1016/ S0002-9378(97)70326-0.

14. Dasari P, Niveditta G, Raghavan S. The maximal vertical pocket and amniotic fluid index in predicting fetal distress in prolonged pregnancy. Int $\mathrm{J}$ Obstet and Gynecol 2007:96;89-93. https://doi.org/10.1016/j.ijgo.2006.09.034. PMID: 17240379.

15. Miyamura $\mathrm{T}$, Masuzaki $\mathrm{H}$, Miyamoto $M$, Ishimaru $\mathrm{T}$. Comparison beween the single deepest pocket and amniotic fluid index in predicting fetal distress in small for gestational age foetuses. Acta Obstet Gynecol Scand. 1997; :123-7. https://doi.org/10.3109/00016349709050066.

16. Alfirevic Z, Lucas M, Walkinshaw SA, McFarlane M, Curran R. A randomized comparison between amniotic fluid index and maximum pool depth in the monitoring of postterm pregnancy. Br J Obstet Gynaecol. 1997;104:20711. https://doi.org/10.1111/j.1471-0528.1997.tb11046.x PMID: 9070140.

17. Magann EF, Chauhan SP, Barrilleaux OS, Whitwoerth NS, Martin JN Jr. Amniotic fluid index and single deepest pocket: weak indicators of abnormal amniotic volumes. Obstet Gynecol. 2000:96:737-40. https:// doi.org/10.1097/00006250-200011000-00017.

18. Moore, Thomas R. Clinical Assessment of Amniotic Fluid.Clinical Obstetrics. 1997;40:303-13. https:// doi.org/10.1097/00003081-199706000-00007.

19. Magann EF, Sandlin AT, Ounpraseuth TS. Amniotic fluid and the clinical relevance of the sonographically estimated amniotic fluid volume. J Ultrasound Med. 2011 Nov;30 (11):1573-85 jum.2011.30.11.1573. PMID: 22039031. 